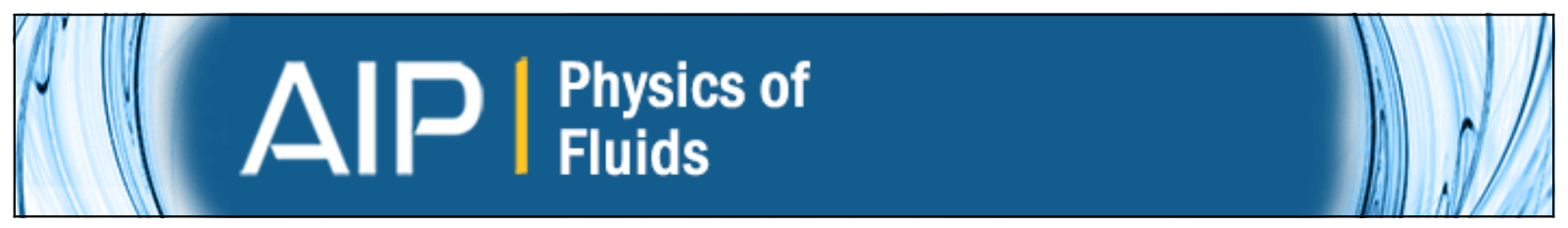

\title{
Capillary-gravity wave drag
}

Shu-Ming Sun and Joseph B. Keller

Citation: Physics of Fluids (1994-present) 13, 2146 (2001); doi: 10.1063/1.1384889

View online: http://dx.doi.org/10.1063/1.1384889

View Table of Contents: http://scitation.aip.org/content/aip/journal/pof2/13/8?ver=pdfcov

Published by the AIP Publishing

\section{Articles you may be interested in}

Capillary-gravity waves generated by a sudden object motion

Phys. Fluids 22, 052107 (2010); 10.1063/1.3430004

Three-dimensional capillary-gravity waves generated by a moving disturbance

Phys. Fluids 19, 082102 (2007); 10.1063/1.2750293

Edge capillary-gravity waves on a sloping beach

Phys. Fluids 17, 048103 (2005); 10.1063/1.1879052

The viscous damping of capillary-gravity waves in a brimful circular cylinder

Phys. Fluids 14, 1910 (2002); 10.1063/1.1476306

Experiments on capillary-gravity waves of solitary type on deep water

Phys. Fluids 9, 1963 (1997); 10.1063/1.869315

\section{AlP Re-register for Table of Content Alerts}

\section{Create a profile.




\title{
Capillary-gravity wave drag
}

\author{
Shu-Ming Sun \\ Department of Mathematics, Virginia Polytechnic Institute and State University, Blacksburg, \\ Virginia 24061-0123 \\ Joseph B. Keller \\ Departments of Mathematics and Mechanical Engineering, Stanford University, Stanford, \\ California 94305-2125
}

(Received 3 January 2001; accepted 3 May 2001)

Drag due to the production of capillary-gravity waves is calculated for an object moving along the surface of a liquid. Both two and three dimensional objects, moving at large Froude and Weber numbers, are treated. (C) 2001 American Institute of Physics. [DOI: 10.1063/1.1384889]

\section{INTRODUCTION AND SUMMARY}

An object $B$, partially submerged in a liquid and moving along its surface, produces capillary-gravity waves which exert a wave drag $R$ on $B$. See Fig. 1. We calculate $R$ when $B$ moves with constant velocity $U$ and is just slightly submerged. Then we indicate how to apply the result to an insect or other arthropod walking on water.

Our calculation applies to a liquid in potential flow with linearized boundary conditions and large values of the Froude number $F=U^{2} / g L$ and the Weber number $W$ $=\rho L U^{2} / T$. Here $\rho$ is the liquid density, $T$ is the surface tension, $g$ is the acceleration of gravity, and $L$ is the scale length of $B$. We determine the wave pattern in both two dimensions (2D) and three dimensions (3D), and show that far from $B$ it is the same as that produced by a moving pressure distribution concentrated along a line in $2 \mathrm{D}$ or at a point in 3D.

The total downward force $P$ in 3D, or force $P$ per unit length in $2 \mathrm{D}$, exerted on the liquid by the equivalent pressure distribution, can be written as

$$
\begin{aligned}
& P=\frac{1}{2} C_{L} \rho U^{2} L^{2} \quad \text { (3D), } \\
& P=\frac{1}{2} C_{L} \rho U^{2} L \quad \text { (2D). }
\end{aligned}
$$

Here $C_{L}$ is a lift coefficient which we find in terms of the body shape, $\frac{1}{2} \rho U^{2}$ is the dynamic pressure, $\pi L^{2}$ is the wetted area of $B$ in $3 \mathrm{D}$, and $2 L$ is the wetted area per unit length in 2D. This downward force must be exerted on $B$ by some external influence, and it is balanced by the lift force exerted on $B$ by the liquid.

$R$ for a moving concentrated pressure distribution was calculated by Raphael and de Gennes. ${ }^{1}$ Their result in $3 \mathrm{D}$ is, when $F W \gg 1$,

$$
R \sim \frac{2 P^{2} \rho U^{2}}{3 \pi T^{2}} \quad(3 \mathrm{D}) .
$$

Their $2 \mathrm{D}$ result is, when $F \gg 1$ and $W \gg 1$,

$$
R \sim \frac{P^{2}}{T} \quad(2 \mathrm{D}) .
$$

We use (1.1) for $P$ in (1.3) for $R$ to get the 3D result

$$
R \sim \frac{C_{L}^{2} \rho^{3} U^{6} L^{4}}{6 \pi T^{2}} \quad(3 \mathrm{D}) .
$$

$C_{L}$ is given by (5.13) for a 3D object with a circular waterline. In $2 \mathrm{D}$ we use (1.2) for $P$ in (1.4) to get

$$
R \sim \frac{C_{L}^{2} \rho^{2} U^{4} L^{2}}{4 T} \quad(2 \mathrm{D}) .
$$

For a $2 \mathrm{D}$ object $C_{L}$ is given by (3.14).

In addition to the lift force $P$ due to dynamic pressure, there are forces on $B$ due to hydrostatic pressure and due to surface tension acting at the waterline. When $B$ is at rest, the resultant of the latter two forces is vertical, and it is just equal to the weight of the fluid displaced by $B$ and by the meniscus $\left(\right.$ Keller $\left.^{2}\right)$. When $B$ is moving, the waterline and these two forces change. To calculate this change requires a boundary layer solution in a neighborhood $M$ of the waterline, which we shall not consider. Within $M$ linearization about a horizontal surface will not be valid if the contact angle condition requires that the surface have a finite slope there.

Raphael and de Gennes ${ }^{1}$ also found that $R=0$ when $U$ $<c_{\min }=(4 g T / \rho)^{1 / 4}$, corresponding to $F W / 4<1$, and that $R$ $=\infty$ at $U=c_{\min }$. Richard and Raphael ${ }^{3}$ showed that including viscosity makes $R>0$ for $U<c_{\text {min }}$ and makes $R$ finite at $U=c_{\min }$. Shliomis and Steinberg ${ }^{4}$ showed that even with $T$ $=0$, for a pressure distribution which extends to infinity in a special way, there is a critical velocity $U_{c}$ such that $R=0$ for $U<U_{c}$.

In Sec. II we formulate the equivalent flow problem of a stationary object in a moving fluid. To solve it asymptotically when $F$ and $W$ are large, we introduce inner and outer asymptotic solutions. In Sec. III we find the 2D inner solution. In Sec. IV we give the 2D outer solution and match it to 


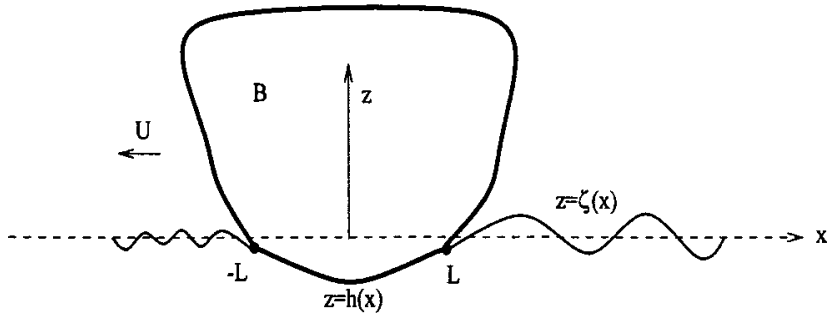

FIG. 1. Sketch of a cross section of the partially submerged body $B$ in two dimensions. Its wetted surface is $z=h(x),-L<x<L$, and the free surface is $z=\zeta(x), x<-L$ and $x>L$. The body is moving to the left with velocity $U$.

the inner solution found in Sec. III. Matching determines $C_{L}$ and $P$, which we use in (1.4) or (1.6) to get $R$ in 2D. In Sec. $\mathrm{V}$ we find the $3 \mathrm{D}$ inner solution and determine $C_{L}$ and $P$. We use them in (1.3) or (1.5) to find $R$ in 3D. In Sec. VI we discuss the application of the results to insects or other arthropods walking on water.

\section{FORMULATION}

We consider a stationary object $B$ partially submerged in a fluid which is in steady potential flow. Far from the object, the fluid has the velocity $-U$ parallel to the $x$ axis, and its surface is $z=0$. We write the potential as $U L[\varphi(x, y, z)$ $-x$ ] where $L$, the half-length of $B$ along the $x$ axis, is the unit of length. The fluid is bounded above by the surface of $B$, $z=h(x, y)$, and by the free surface $z=\zeta(x, y)$ outside $B$. It is convenient to denote by $H$ the projection on the plane $z=0$ of the wetted surface of $B$, and by $H^{C}$ the rest of the plane, which is the projection of the free surface. The common boundary of $H$ and $H^{C}$ is the projection on $z=0$ of the waterline, the neighborhood $M$ of which contains the meniscus.

We assume that outside $M$ both $h(x, y)$ and $\zeta(x, y)$ and their derivatives are sufficiently small that the boundary conditions can be linearized and imposed on the plane $z=0$. Then the equations satisfied by $\varphi$ and $\zeta$ there are

$$
\begin{aligned}
& \Delta \varphi=0, \quad z<0, \\
& \varphi_{z}=h_{x}, \quad z=0,(x, y) \in H, \\
& \varphi_{z}=\zeta_{x}, \quad z=0,(x, y) \in H^{C}, \\
& \varphi_{x}+F^{-1} \zeta-W^{-1}\left(\zeta_{x x}+\zeta_{y y}\right)=0, \quad z=0,(x, y) \in H^{C}, \\
& \nabla \varphi \rightarrow 0, \quad z \rightarrow-\infty .
\end{aligned}
$$

The solution must contain only outgoing waves, and must match with the solution in $M$, where these equations do not hold. In (2.4), $F=U^{2} / g L$ is the Froude number and $W$ $=\rho L U^{2} / T$ is the Weber number.

We shall solve this problem when both $F$ and $W$ are large. Then we keep only the leading term $\varphi_{x}$ in (2.4), omitting the terms in $F^{-1}$ and $W^{-1}$, to obtain the simplified boundary condition

$$
\varphi_{x}=0, \quad z=0, \quad(x, y) \in H^{C} .
$$

We call the solution of the problem with (2.6) replacing (2.4) the "inner solution." It is the leading term in an asymptotic

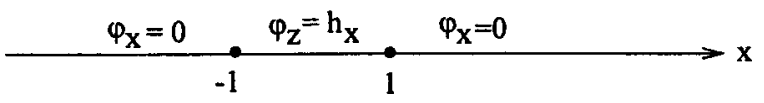

FIG. 2. The inner solution in 2D satisfies $\Delta \varphi=0$ in the half-plane $z<0$ with the boundary conditions shown on the line $z=0$.

expansion with respect to $F$ and $W$ for $F$ and $W$ large. Since (2.6) does not involve $\zeta$, Eqs. (2.1), (2.2), (2.5), and (2.6) can be solved for $\varphi$ and then $\zeta$ can be found from $\varphi$ by integrating (2.3).

The inner solution can be constructed in the entire fluid region $z \leqslant 0$ without the necessity of matching it to a boundary layer solution in $M$ and without imposing the outgoing wave condition. This is so because (2.6) does not contain derivatives of $\zeta$, so no contact angle condition is required at the waterline, and (2.6) cannot support surface waves. Instead of the contact angle condition, it suffices to require the solution to have the weakest singularity at the waterline. Instead of the radiation or outgoing wave condition, it suffices to require that the velocity $\boldsymbol{\nabla} \varphi$ tend to zero at infinity. These two requirements select a unique solution.

The inner solution does not contain surface waves, so it is not valid far from $B$, where the waves propagate. Therefore, we shall introduce another solution, which we call the "outer solution," which is valid there but not valid near $B$. Since it is not valid near $B$, we do not require that it satisfy the condition (2.2) on $B$. Instead, we introduce a dimensionless pressure distribution on the right-hand side of (2.4) to produce the waves contained in the outer solution. This pressure distribution, which is unknown, depends upon $F$ and $W$. We assume that it can be expanded asymptotically in $F$ and $W$ for $F$ and $W$ large, and we denote by $p(x, y)$ the leading term in it. To determine $p(x, y)$ we require that the outer solution match with the inner solution at some intermediate distance from $B$ where both are valid.

The problem for the leading term in the outer solution is obtained by replacing (2.2) and (2.4) by the single equation

$$
\varphi_{x}+F^{-1} \zeta-W^{-1}\left(\zeta_{x x}+\zeta_{y y}\right)=-p(x, y), \quad z=0 .
$$

The radiation condition must still hold, but there is no condition at $B$. We can eliminate $\zeta$ by taking the $x$ derivative of (2.7) and using (2.3) to replace $\zeta_{x}$ by $\varphi_{z}$. Then (2.7) becomes

$$
\varphi_{x x}+F^{-1} \varphi_{z}-W^{-1}\left(\varphi_{z x x}+\varphi_{z y y}\right)=-p_{x}(x, y), \quad z=0 .
$$

Now (2.1), (2.5), (2.8) and the radiation condition constitute a problem for $\varphi$ alone. Then $\zeta$ can be found from $\varphi$ by integrating (2.3).

\section{INNER SOLUTION IN TWO DIMENSIONS}

We begin by considering a two dimensional body $B$ with surface $z=h(x)$, and the corresponding two dimensional flow. The potential $\varphi$ of the inner solution satisfies (2.1), (2.2), (2.6) and the condition $\nabla \varphi \rightarrow 0$ at infinity. See Fig. 2. It is convenient to set $u=\varphi_{x}$, and to write these equations in terms of $u$ : 


$$
\begin{aligned}
& u_{x x}+u_{z z}=0, \quad z \leqslant 0, \\
& u_{z}=h_{x x}(x), \quad z=0, \quad|x|<1, \\
& u=0, \quad z=0, \quad|x|>1 .
\end{aligned}
$$

Since the wetted part of $B$ has length $2 L$ along the $x$ axis, we have placed the origin at its midpoint. Then in the scaled variables it extends from $x=-1$ to $x=+1$. The solution $u$ must tend to zero at infinity.

To solve for $u$ we introduce the complex variable $w=x$ $+i z$ and map the lower half of the $w$ plane onto a half strip in the plane of $\lambda=\xi+i \eta$ by setting $w=\sin \lambda$. Then

$$
x=\sin \xi \cosh \eta, \quad z=\cos \xi \sinh \eta .
$$

In the $\lambda$-plane $u(\xi, \eta)$ satisfies

$$
\begin{aligned}
& u_{\xi \xi}+u_{\eta \eta}=0, \quad-\frac{\pi}{2}<\xi<\frac{\pi}{2}, \quad \eta<0, \\
& u_{\eta}=h_{x x}(\sin \xi) \cos \xi, \quad-\frac{\pi}{2}<\xi<\frac{\pi}{2}, \quad \eta=0, \\
& u=0, \quad \xi= \pm \frac{\pi}{2}, \quad \eta<0 .
\end{aligned}
$$

The solution can be found by separation of variables as a sum of products of trigonometric functions of $\xi$ multiplied by functions of $\eta$. The normalized functions of $\xi$ which satisfy (3.7) are $(2 / \pi)^{1 / 2} \sin [n(\xi-\pi / 2)], n=1,2, \ldots$. For each $n$ the corresponding function of $\eta$ is a linear combination of $e^{n \eta}$ and $e^{-n \eta}$, but the requirement that $u \rightarrow 0$ as $\eta \rightarrow-\infty$ shows that only the $e^{n \eta}$ are present. Thus we can write $u$ as

$$
u(\xi, \eta)=\sum_{n=1}^{\infty} a_{n} e^{n \eta} \sin \left[n\left(\xi-\frac{\pi}{2}\right)\right] .
$$

From (3.6) we find that $a_{n}$ is given by

$$
a_{n}=\frac{2}{n \pi} \int_{-\pi / 2}^{\pi / 2} h_{x x}(\sin \xi) \cos \xi \sin \left[n\left(\xi-\frac{\pi}{2}\right)\right] d \xi .
$$

For $n=1$ we can simplify this integral since $\sin [\xi-(\pi / 2)]$ $=-\cos \xi$. By using this, and setting $s=\sin \xi$, we obtain

$$
a_{1}=-\frac{1}{\pi} \int_{-1}^{1} h_{x x}(s) \sqrt{1-s^{2}} d s
$$

To find the behavior of $u$ for $x^{2}+z^{2}$ large, with $z$ negative, we first invert (3.4) to get

$$
\begin{aligned}
& e^{\eta} \sim 1 / 2 \sqrt{x^{2}+z^{2}}, \quad \cos \xi \sim|z| / \sqrt{x^{2}+z^{2}}, \\
& \sin \xi \sim x / \sqrt{x^{2}+z^{2}} .
\end{aligned}
$$

Then from (3.8) we have

$$
u \sim-a_{1} e^{\eta} \cos \xi=\frac{a_{1} z}{2\left(x^{2}+z^{2}\right)}, \quad z<0, \quad x^{2}+z^{2} \gg 1 .
$$

The dimensionless dynamic pressure in the flow is, from the Bernoulli equation,

$$
-\frac{1}{2}\left[\left(-1+\varphi_{x}\right)^{2}+\varphi_{y}^{2}-1\right]=\varphi_{x}+O\left[(\nabla \varphi)^{2}\right] .
$$

In the linear approximation, this pressure is just $\varphi_{x}=u$. Therefore on $B$ we have

$$
p(x)=u(\xi, 0)=\sum_{n=1}^{\infty} a_{n} \sin \left[n\left(\xi-\frac{\pi}{2}\right)\right], \quad x=\sin \xi .
$$

We can use $p(x)$ in (2.7) to calculate the outer solution. We can also integrate (3.13) to get $C_{L}$, the lift coefficient or total dimensionless dynamic lift force per unit length, on $B$. Since $x=\sin \xi$ on $B$ and $a_{1}$ is given by (3.10), we obtain

$$
\begin{aligned}
C_{L} & =2 \int_{-1}^{1} p(x) d x \\
& =2 a_{1} \int_{-1}^{1}(-\cos \xi) d x \\
& =-\pi a_{1} \\
& =\int_{-1}^{1} h_{x x}(s) \sqrt{1-s^{2}} d s .
\end{aligned}
$$

Next from (2.3) we have $\zeta_{x}(x)=\varphi_{z}(x, 0)$ so $\zeta_{x x}(x)$ $=\varphi_{x z}(x, 0)=u_{z}(x, 0)$. From (3.12) we get $u_{z}(x, 0) \sim a_{1} / 2 x^{2}$ and therefore $\zeta_{x x}(x) \sim a_{1} / 2 x^{2}$. Integrating yields $\zeta_{x}$ $\sim-a_{1} / 2 x$, where we have taken the constant of integration to be zero to make $\zeta_{x}(\infty)=0$. Another integration gives

$$
\zeta(x) \sim-\frac{a_{1}}{2} \log |x|+A_{ \pm} .
$$

The constant is $A_{+}$for $x>0$ and $A_{-}$for $x<0$. This result shows clearly that the inner solution is not valid for large $x$ since it yields a free surface which is unbounded at infinity.

\section{OUTER SOLUTION IN TWO DIMENSIONS}

The outer solution, which satisfies (2.1), (2.5), (2.8) and the radiation condition, can be found for any $p(x)$ by using the Fourier transform in $x$. For $p(x)=P \delta(x)$, the surface $\zeta(x)$ is given explicitly by Raphael and de Gennes. ${ }^{1}$ In our notation it is

$\zeta(x)=\frac{-2 P}{T\left(k_{2}-k_{1}\right)} \sin k_{j} x+F(x), \quad \begin{array}{ll}k_{j}=k_{1} \text { for } x<0, \\ k_{j}=k_{2} \text { for } x>0 .\end{array}$

The wave numbers $k_{1}$ and $k_{2}$ are defined by

$k_{1}=\frac{1}{2} W-\frac{1}{2}\left(W^{2}-4 W F^{-1}\right)^{1 / 2} \sim F^{-1}$

$k_{2}=\frac{1}{2} W+\frac{1}{2}\left(W^{2}-4 W F^{-1}\right)^{1 / 2} \sim W$

for $W \gg 1, \quad F \gg 1$.

Thus there are gravity waves with wave number $k_{1} \sim F^{-1}$ downstream of $B$ and capillary waves with wave number $k_{2} \sim W$ upstream of $B$. The nonoscillatory function $F(x)$ is defined by

$$
F(x)=\frac{P}{\pi T\left(k_{2}-k_{1}\right)} \int_{0}^{\infty}\left(\frac{m}{m^{2}+k_{1}^{2}}-\frac{m}{m^{2}+k_{2}^{2}}\right) e^{-m|x|} d m .
$$

For $W$ and $F$ large, the integral in (4.3) can be evaluated asymptotically. The integral of the first term can be written as follows by setting $m|x|=t$, and then evaluated for $k_{1}$ small: 


$$
\begin{aligned}
\int_{0}^{\infty} \frac{t e^{-t} d t}{t^{2}+\left(k_{1} x\right)^{2}}= & \int_{0}^{1} \frac{t d t}{t^{2}+\left(k_{1} x\right)^{2}} \\
& +\int_{0}^{1} \frac{t\left(e^{-t}-1\right)}{t^{2}+\left(k_{1} x\right)^{2}}+\int_{1}^{\infty} \frac{t e^{-t} d t}{t^{2}+\left(k_{1} x\right)^{2}} \\
= & -\log k_{1}|x|+\frac{1}{2} \log \left[1+\left(k_{1} x\right)^{2}\right]+O(1)
\end{aligned}
$$

This result is obtained by evaluating the first integral, and noting that both the second and third integrals are finite when $k_{1} x=0$. The integral of the second term can be estimated for $\left|k_{2} x\right|$ large by writing

$$
\int_{0}^{\infty} \frac{t e^{-t} d t}{t^{2}+\left(k_{2} x\right)^{2}}<\frac{1}{\left(k_{2} x\right)^{2}} \int_{0}^{\infty} t e^{-t} d t=\frac{1}{\left(k_{2} x\right)^{2}} .
$$

We now combine (4.4) and (4.5) in (4.3) and use (4.2) for $k_{1}$ and $k_{2}$ to get $T\left(k_{2}-k_{1}\right) \sim T W=\rho U^{2} L$. Then (4.3) yields

$$
F(x) \sim \frac{P}{\pi \rho U^{2} L}\left[-\log |x|-\log k_{1}+O\left(\frac{1}{\left(k_{2} x\right)^{2}}\right)+O(1)\right] .
$$

Next we use (4.6) in (4.1) for $\zeta(x)$, and compare the result with the inner solution (3.15). We see that the leading terms are both proportional to $\log |x|$. The two coefficients of $\log |x|$ are equal if $P /\left(\frac{1}{2} \rho U^{2} L\right)$ has the value

$$
\frac{P}{\frac{1}{2} \rho U^{2} L}=\pi a_{1} \text {. }
$$

This is exactly the negative of the lift coefficient $C_{L}$ given by (3.14).

This analysis confirms that the pressure distribution $P \delta(x)$, with $P$ the negative of the force on $B$ due to dynamic pressure, produces an outer solution which matches the inner solution. Therefore this value of $P$ can be used in (1.4), or $C_{L}$ given in (3.14) can be used in (1.6), to determine $R$, the wave drag per unit length of $B$ :

$$
R \sim \frac{\rho^{2} U^{4} L^{2}}{4 T}\left[\int_{-1}^{1} h_{x x}(x)\left(1-x^{2}\right)^{1 / 2} d x\right]^{2}
$$

By using $P$ in (4.1) we find that the dimensionless amplitude of both the gravity and capillary waves is $-\pi a_{1}$, so the actual amplitude is $-\pi a_{1} L=L \int_{-1}^{1} h_{x x}(x)\left(1-x^{2}\right)^{1 / 2} d x$.

\section{INNER SOLUTION IN THREE DIMENSIONS}

To find the inner solution for a three dimensional body $B$, we again set $\varphi_{x}=u$ in (2.1), (2.2), and (2.6) to get

$$
\begin{aligned}
& \Delta u=0, \quad z=0, \\
& u_{z}=h_{x x}(x, y), \quad z=0,(x, y) \in H, \\
& u=0, \quad z=0,(x, y) \in H^{C} .
\end{aligned}
$$

In addition, $u \rightarrow 0$ at infinity.

Now to facilitate solving this problem, we take the waterline projection to be the unit circle so that $H$ is the unit disk. Then we introduce oblate spheroidal coordinates $\xi, \eta, \varphi$ with the ranges $\xi \in(0,+\infty), \eta \in(-1,0)$, and $\varphi \in(0,2 \pi)$, related to $x, y, z$ by

$$
\begin{aligned}
& x=\left(\xi^{2}+1\right)^{1 / 2}\left(1-\eta^{2}\right)^{1 / 2} \cos \varphi, \\
& y=\left(\xi^{2}+1\right)^{1 / 2}\left(1-\eta^{2}\right)^{1 / 2} \sin \varphi, \quad z=\xi \eta .
\end{aligned}
$$

In these coordinates, Laplace's equation (5.1) is separable with product solutions $\Phi(\varphi) P(\eta) Q(\xi)$ and (5.2) and (5.3) become

$$
\begin{aligned}
& u=0 \quad \text { at } \eta=0, \\
& u_{\xi}=\eta h_{x x}\left[\left(1-\eta^{2}\right)^{1 / 2} \cos \varphi,\left(1-\eta^{2}\right)^{1 / 2} \sin \varphi\right] \text { at } \xi=0 .
\end{aligned}
$$

The function $\Phi$ is a linear combination of $\sin m \phi$ and $\cos m \phi$, where $m$ must be an integer to make $\Phi$ periodic with period $2 \pi$. The function $P$ is a linear combination of the associated Legendre functions $P_{n}^{m}(\eta)$ and $Q_{n}^{m}(\eta)$. However, $Q_{n}^{m}$ must be omitted because it is singular in the domain, and $n$ must be an integer of the form $n=m+2 \ell+1, \ell=0,1, \ldots$ in order that $P_{n}^{m}(0)=0$ as $(5.5)$ requires. Finally, $Q$ is a linear combination of $P_{n}^{m}(i \xi)$ and $Q_{n}^{m}(i \xi)$, but $P_{n}^{m}(i \xi)$ is singular at $\xi=+\infty$, so it must be omitted.

By combining these product solutions, we can write the general solution of (5.1) and (5.5), and the condition $u \rightarrow 0$ at infinity in the form

$$
\begin{aligned}
u(\xi, \eta, \varphi)= & \sum_{\ell=0}^{\infty} \sum_{m=0}^{\infty}\left(A_{m \ell} \cos m \varphi\right. \\
& \left.+B_{m \ell} \sin m \varphi\right) P_{m+2 \ell+1}^{m}(\eta) Q_{m+2 \ell+1}^{m}(i \xi) \\
& \times\left[\left.\frac{d}{d \xi} Q_{m+2 \ell+1}^{m}(i \xi)\right|_{\xi=0}\right]^{-1}
\end{aligned}
$$

We have included the last factor for convenience. The derivative in it has the value

$$
\begin{aligned}
\lim _{\xi \rightarrow 0} \frac{d}{d \xi} Q_{m+2 \ell+1}^{m}(i \xi) & =-(2 \ell+2) i \lim _{\xi \rightarrow 0} Q_{m+2 \ell+2}^{m}(i \xi) \\
& =\frac{-e^{i m \pi / 2} \pi(-1)^{m+\ell+1}}{2^{m+2 \ell+1} \ell !(m+\ell) !}
\end{aligned}
$$

The product $e^{-i m \pi / 2} Q_{m+2 l+1}^{m}(i \xi)$ is real for $\xi \geqslant 0$.

We substitute (5.7) into (5.6) to get

$$
\begin{aligned}
\sum_{\ell=0}^{\infty} & \sum_{m=0}^{\infty}\left(A_{m \ell} \cos m \varphi+B_{m \ell} \sin m \varphi\right) P_{m+2 \ell+1}^{m}(\eta) \\
\quad & =\eta h_{x x}\left[\left(1-\eta^{2}\right)^{1 / 2} \cos \varphi,\left(1-\eta^{2}\right)^{1 / 2} \sin \varphi\right] \\
& \equiv g(\eta, \varphi) .
\end{aligned}
$$

Here we have defined $g(\eta, \varphi)$ as the expression $\eta h_{x x}$ in (5.9). By using the orthogonality of the trigonometric functions and of the associated Legendre functions in (5.9), we obtain 


$$
\begin{aligned}
\left(\begin{array}{l}
A_{m \ell} \\
B_{m \ell}
\end{array}\right)= & \frac{\varepsilon_{m}}{2 \pi} \frac{(2 m+4 \ell+2+1)(2 \ell+1) !}{(2 m+2 \ell+1) !} \\
& \times \int_{0}^{2 \pi}\left(\begin{array}{c}
\cos m \varphi \\
\sin m \varphi
\end{array}\right) \int_{-1}^{0} g(\eta, \varphi) P_{m+2 \ell+1}^{m}(\eta) d \eta d \varphi .
\end{aligned}
$$

The factor $\varepsilon_{m}=2$ for $m \geqslant 1$ and $\varepsilon_{0}=1$. With this determination of the coefficients the solution $u(\xi, \eta, \varphi)$ is determined by (5.7).

The expression (5.10) for $A_{00}$ can be simplified as follows, since $P_{1}^{0}(\eta)=\eta$ :

$$
\begin{aligned}
A_{00}= & \frac{3}{2 \pi} \int_{0}^{2 \pi} \int_{-1}^{0} h_{x x}\left[\left(1-\eta^{2}\right)^{1 / 2}\right. \\
& \left.\times \cos \varphi,\left(1-\eta^{2}\right)^{1 / 2} \sin \varphi\right] \eta^{2} d \eta d \varphi \\
= & \frac{3}{2 \pi} \int_{0}^{2 \pi} \int_{0}^{1} h_{x x}[r \cos \varphi, r \sin \varphi]\left(1-r^{2}\right)^{1 / 2} r d r d \varphi \\
= & \frac{3}{2 \pi} \iint_{x^{2}+y^{2}<1} h_{x x}(x, y)\left[1-x^{2}-y^{2}\right]^{1 / 2} d x d y .
\end{aligned}
$$

The dimensionless dynamic pressure on the wetted surface of $B$ is just $u(0, \eta, \varphi)$ for $-1 \leqslant \eta \leqslant 0$, so it is given by (5.7) with $\xi=0$. By integrating it over this wetted surface, i.e., over the disk $-1 \leqslant \eta \leqslant 0$, we get $C_{L}$, the lift coefficient or dimensionless lift force. The area element is $r d r d \varphi$ $=\left(1-\eta^{2}\right)^{1 / 2} d\left(1-\eta^{2}\right)^{1 / 2} d \varphi=-\eta d \eta d \varphi$. The $\varphi$ integration yields zero for all terms in the sum except the cosine terms with $m=0$. Then the remaining integrands are constants times $\eta P_{2 \ell+1}^{0}(\eta)$. The integral of each of these terms from $\eta=-1$ to $\eta=0$ vanishes, except for the term with $\ell=0$, which is $-1 / 3$. Thus we obtain

$$
\begin{aligned}
C_{L}= & 2 \int_{0}^{2 \pi} \int_{-1}^{0} u(0, \eta, \varphi)(-\eta) d \eta d \varphi \\
= & 4 \pi A_{00} \int_{-1}^{0} P_{1}^{0}(\eta)(-\eta) d \eta Q_{1}^{0}(0) \\
& \times\left[\left.\frac{d}{d \xi} Q_{1}^{0}(i \xi)\right|_{\xi=0}\right]^{-1}=\frac{8}{3} A_{00} .
\end{aligned}
$$

When we use (5.11) for $A_{00}$ in (5.12), we get

$$
C_{L}=\frac{4}{\pi} \iint_{x^{2}+y^{2}<1} h_{x x}(x, y)\left(1-x^{2}-y^{2}\right)^{1 / 2} d x d y .
$$

The result (5.13) for $C_{L}$ can be used in (1.1) and (1.5) to determine $P$ and $R$ in 3D.

We can also calculate $u$ for $x^{2}+y^{2}+z^{2} \gg 1$ from (5.7) by using the inversion of the coordinate transformation (5.4) to get

$$
\begin{array}{r}
\xi \sim\left(x^{2}+y^{2}+z^{2}\right)^{1 / 2}, \quad \eta \sim z\left(x^{2}+y^{2}+z^{2}\right)^{-1 / 2} \\
\text { for } x^{2}+y^{2}+z^{2} \gg 1 .
\end{array}
$$

Furthermore $Q_{m+2 \ell+1}^{m}(i \xi)=O\left(\xi^{-2 \ell-m-2}\right) \quad$ as $\quad \xi \rightarrow \infty$. Therefore the leading term in (5.7) is that with $\ell=m=0$ :

$$
\begin{aligned}
u(x, y, z) & \sim \frac{2 A_{00}}{\pi} P_{1}^{0}(\eta) Q_{1}^{0}(i \xi) \\
& \sim \frac{2 A_{00} \eta}{\pi}\left(\frac{-1}{3 \xi^{2}}\right)=\frac{-2 A_{00}}{3 \pi} \frac{z}{\left(x^{2}+y^{2}+z^{2}\right)^{3 / 2}} .
\end{aligned}
$$

We have verified that the outer solution, corresponding to the pressure $P \delta(x) \delta(y)$, agrees asymptotically with (5.15) when $P /\left(\frac{1}{2} \rho U^{2} L^{2}\right)$ is given by (5.13).

\section{WALKING ON WATER}

An insect or other arthropod walking on water is supported mainly by surface tension acting on its partly submerged legs. In walking, it keeps some of its legs fixed with respect to its body, and moves the others backward. Then it brings those legs forward, presumably lifting them to diminish drag. It repeats the process, moving the same or other legs backward. The propulsive force is provided by the resistance of the water to the backward motion of the backward moving legs. This must exceed the resistance of the water to the forward motion of the fixed legs, and to the forward motion of those legs being brought forward.

The total drag force on a moving leg is a sum $R+R_{f}$ $+R_{T}$ where $R$ is the wave-making drag, $R_{f}$ is the viscous drag, also called the drag due to skin friction, and $R_{T}$ is the drag due to surface tension. When $U \leqslant c_{\min }=2^{1 / 2}(g T / \rho)^{1 / 4}$, where $c_{\min }$ is the minimum phase velocity of capillarygravity waves, there are no waves so $R=0$. As $U$ increases above $c_{\min }, R$ increases from zero and it is asymptotic to the expression in (1.5) when $U$ is large. Thus the total drag is given by $R_{f}+R_{T}$ for $U \leqslant c_{\min }$, increases above this value as $U$ increases above $c_{\min }$, and is asymptotic to the expression in (1.5) for large $U$ because that expression increases faster than $R_{f}+R_{T}$.

Measurements of the total drag on the leg of the fisher spider were made by Suter et al..$^{5}$ for $U$ ranging from zero to about $2 c_{\min }$. In walking, $U$ ranges up to $3 c_{\min }$. They observed the presence of waves for $U>c_{\text {min }}$, but they were unable to measure their effect on the total drag. A calculation like the present one, valid for $U$ up to about $2 c_{\min }$ or $3 c_{\min }$, would be helpful in separating the total drag into its components.

\section{ACKNOWLEDGMENTS}

S.-M.S. was supported in part by the National Science Foundation and J.B.K. was supported in part by the Air Force Office of Scientific Research.

\footnotetext{
${ }^{1}$ E. Raphael and P.-G. de Gennes, "Capillary gravity waves caused by a moving disturbance; Wave resistance," Phys. Rev. E 53, 3448 (1996).

${ }^{2}$ J. B. Keller, "Surface tension force on a partially submerged body," Phys. Fluids 10, 3009 (1998).
} 
${ }^{3}$ D. Richard and E. Raphaël, "Capillary-gravity waves: The effect of viscosity on the wave resistance," Europhys. Lett. 48, 49 (1999).

${ }^{4}$ M. I. Shliomis and V. Steinberg, "Surface gravity waves due to a moving body: Onset of wave resistance as a critical phenomenon," Phys. Rev.
Lett. 79, 4178 (1997)

${ }^{5}$ R. B. Suter, O. Rosenberg, S. Loeb, H. Wildman, and J. H. Long, Jr., "Locomotion on the water surface-propulsive mechanisms of the fisher spider Dolomedes triton,” J. Exp. Biol. 200, 2523 (1997). 